\title{
Preparation and Characterization of Water-based Magnetic Fluids
}

\author{
Zuocheng DAI ${ }^{\mathrm{a}}$, Yan $\mathrm{HUANG}^{\mathrm{b}}$, Huan YANG ${ }^{\mathrm{c}}$, Yicheng LIU $^{\mathrm{d}}$ and Yuxiang YANG ${ }^{\mathrm{e}}{ }^{*}$ \\ School of Chemistry and Molecular Engineering, East China University of Science and Technology, \\ Shanghai 200237, China \\ a13501822836@163.com b82204318@qq.com, '261474877@qq.com, d271980853@qq.com, \\ eyxyang@ecust.edu.cn
}

\begin{abstract}
Water-based magnetic fluids $\left(\mathrm{Fe}_{3} \mathrm{O}_{4}\right)$ with good dispersion were synthesized by co-precipitation method in this study. The synthesis conditions for the product were studied and results showed that the particle sizes were between $10-20 \mathrm{~nm}$, and the nanoparticles proved to be Nano $\mathrm{Fe}_{3} \mathrm{O}_{4}$. Stability tests for the water-based magnetic fluids showed that the product had better dispersion both in ethanol and water, using the PEG-4000 as the surfactant. The nanoparticles can be placed in the air for a long time, which is good for preparation of core-shell composite materials when needed.
\end{abstract}

\section{Introduction}

Magnetic fluid is a highly stable colloidal solution ${ }^{[1]}$ that is made up of ferromagnetic or ferrimagnetic particles which are highly dispersed in liquid carrier.The particle size for the magnetic particles is not more than $20 \mathrm{~nm}$. The liquid carrier can be selected by seal medium and working conditions which can be water, kerosene, or silicone oil ${ }^{[2-6]}$.Because of the special physical properties, chemical properties and fluid properties of water-based magnetic fluids, they can be widely used in mechanical, chemical, medical and other fields ${ }^{[7]}$, especially in hyperthermia.

In this study PEG-4000 was used as the surfactant in the co-precipitation method when preparing the water-based magnetic fluid with good suspension. The magnetic fluid remained stable after being placed in the air for a long time, providing the basis for its further use in the next preparation of core-shell structure composites.

\section{Experimental}

\subsection{Materials}

$\mathrm{FeCl}_{3} \cdot 6 \mathrm{H}_{2} \mathrm{O}, \mathrm{FeCl}_{2} \cdot 4 \mathrm{H}_{2} \mathrm{O}$, polyethylene glycol (PEG-4000), $\mathrm{NaOH}$, and anhydrous ethanol (99\%)were of AR grade. Ethanol (95\%) was of CP grade. All the reagents were

\footnotetext{
*Corresponding Author: yxyang@ecust.edu.cn
} 
produced by Sinopharm Chemical Reagent Co., Ltd. and used as received.

\subsection{Preparation of magnetic fluid}

To prepare magnetic fluid, a mixture of $0.30 \mathrm{~g} \mathrm{FeCl}_{3} \cdot 6 \mathrm{H}_{2} \mathrm{O}, 0.27 \mathrm{~g} \mathrm{FeCl}_{2} \cdot 4 \mathrm{H}_{2} \mathrm{O}$ and $12.5 \mathrm{~g}$ PEG-4000 was added to $60 \mathrm{~mL}$ of boiled distilled water with stirring at room temperature. The stirring was carried through the high-purity nitrogen. When the temperature was raised to $80^{\circ} \mathrm{C}$, the mixed solution of $3.0 \mathrm{~mL} 2.5 \mathrm{~mol} / \mathrm{L} \mathrm{NaOH}$ and $50 \mathrm{~mL}$ $250 \mathrm{~g} / \mathrm{L}$ PEG-4000 was poured into a constant pressure dropping funnel and slowly added drop wise into three-necked flask containing the above mixture. The high-purity nitrogen was continuously purged into this solution and the mixture was stirred at $80{ }^{\circ} \mathrm{C}$ for $3 \mathrm{~h}$. Finally, black precipitates were separated by using magnetic and washed with water. The magnetic fluid was formulated into solutions of different concentrations and stored at a nitrogen atmosphere.

\section{Resluts and Discussion}

\subsection{X-Ray Diffraction}

$\mathrm{X}$-ray diffraction patterns form the no-PEG-coated $\mathrm{Fe}_{3} \mathrm{O}_{4}$ nanoparticles and PEG-modified magnetic fluids are shown in Fig 1. After the XRD patterns contract, we found the magnetic fluid, obtained using the PEG-4000 as a surface active agent after calcinations, with no alteration of the crystallization properties, agreeing well with the $\mathrm{Fe}_{3} \mathrm{O}_{4}$ standard diffraction with the typical peaks at $30.1^{\circ}, 35.5^{\circ}, 43.1^{\circ}, 53.4^{\circ}$, and $57.0^{\circ}$ corresponding to (220), (311), (400), (422), (511) and (440) crystal planes, respectively. The PEG-coated $\mathrm{Fe}_{3} \mathrm{O}_{4}$ nanoparticles exhibited the same characteristic diffraction peaks as pure $\mathrm{Fe}_{3} \mathrm{O}_{4}$. These results indicated that the PEG-coated $\mathrm{Fe}_{3} \mathrm{O}_{4}$ nanoparticles had been successfully synthesized and the crystal structure of the $\mathrm{Fe}_{3} \mathrm{O}_{4}$ did not change after being coated with the PEG.
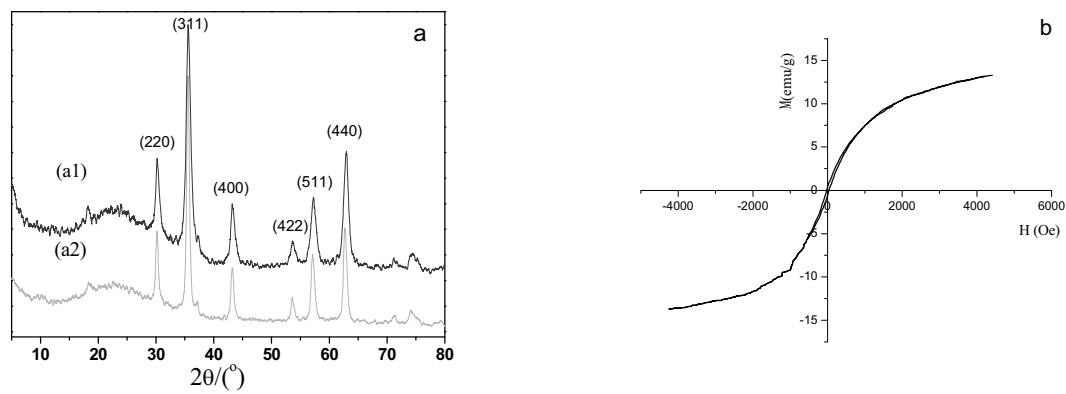

Fig. 1 Characterization of magnetic fluids : (a)XRD pattern of nano- $\mathrm{Fe}_{3} \mathrm{O}_{4}$ particles: (a1) calcined magnetic fluid,(a2) nano- $\mathrm{Fe}_{3} \mathrm{O}_{4}$ particles without modification of PEG-4000,(b) Hysteresis loop of super-paramagnetic nano $\mathrm{Fe}_{3} \mathrm{O}_{4}$ particles 


\subsection{Magnetic Testing}

As shown in Fig 1(b), the hysteresis loop for the magnetic $\mathrm{Fe}_{3} \mathrm{O}_{4}$ nanoparticles was almost not hysteresis, showing superparamagnetic. However, the diameter of the $\mathrm{Fe}_{3} \mathrm{O}_{4}$ nanoparticles was small and so it had low saturation magnetization andthe coercive force was small. The coercive force Hc was $28.11 \mathrm{Oe}$, with saturation magnetization (Ms) of $13.26 \mathrm{emu} / \mathrm{g}$, and residual magnetization (Mr)of $0.45 \mathrm{emu} / \mathrm{g}$.

\subsection{TEM analysis}

Magnetic fluid was prepared by washing three times, followed by drying in vacuo at $80{ }^{\circ} \mathrm{C}$ for 4 hours, and calcined at $500{ }^{\circ} \mathrm{C}$ for $1 \mathrm{~h}$ under nitrogen atmosphere. Fig 2(a) shows TEM image of the sample after calcinations. Fig 2(a)shows the particles had good dispersibility and the size for the nanoparticles was about $10 \mathrm{~nm}$. Different particles were connected through the white matter, which was due to the PEG-4000 coating. During the reaction, the PEG-4000 was coated on the surface of the $\mathrm{Fe}_{3} \mathrm{O}_{4}$ nanoparticles, thereby reducing the surface energy of the nanoparticles and preventing further agglomeration of the particles. After calcination at $500{ }^{\circ} \mathrm{C}$, excess PEG-4000 was removed and there was only a small amount of coating on the particle surface. The particles had good dispersibility after the calcinations.. The solvent layer was formed to prevent the agglomeration of small particles and this may be due to the PEG-4000 coating on the surface of the $\mathrm{Fe}_{3} \mathrm{O}_{4}$ nanoparticles with covalent bond or hydrogen bond. This shows that the PEG-4000 was used as surface coating agent and dispersant in the synthesis of the magnetic fluid.
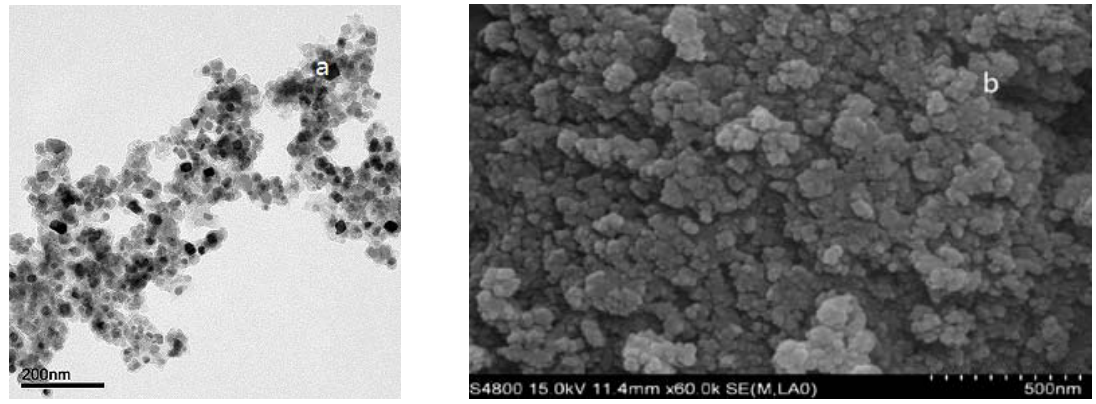

Fig. 2 Morphology analysis of magnetic fluids : (a)TEM image of calcined magnetic fluid, (b)SEM image of calcined magnetic fluid

\subsection{SEM analysis}

Fig 2(b) shows SEM image of calcined magnetic fluid. The Fig. shows the generated nano- $\mathrm{Fe}_{3} \mathrm{O}_{4}$ particles were arranged relatively densely, forming a layered structure. This was because of the surface coating of the particles with PEG-4000. The observed TEM results also coincided with these results.

\subsection{Influence of different concentrations of PEG solution for MHD stability}

Suspension rate was used to characterize the stability of the magnetic fluid. The higher suspension rate illustrated better stability for the magnetic fluid. Test method: $10 \mathrm{~mL}$ of prepared magnetic fluid was put in a graduated cylinder after standing at different times, 
and respectively recorded at scale V under liquid suspension. Suspension rate was calculated as follows:

$$
\text { Suspension rate }=\mathrm{V} / 10 \times 100 \%
$$

In order to find the optimal dosage for PEG-4000, the solution with $150 \mathrm{~g} / \mathrm{L}, 200 \mathrm{~g} / \mathrm{L}$, $250 \mathrm{~g} / \mathrm{L}$ and $300 \mathrm{~g} / \mathrm{L}$ concentration was prepared and its quality was kept and amount of alkali and iron salt unchanged. The solution of magnetic fluid was formulated into $2 \mathrm{mg} / \mathrm{ml}$ concentration. The magnetic fluid suspension rate changes were determined using the method described above at $\mathrm{pH} \approx 7$, under different standing times, as shown below:

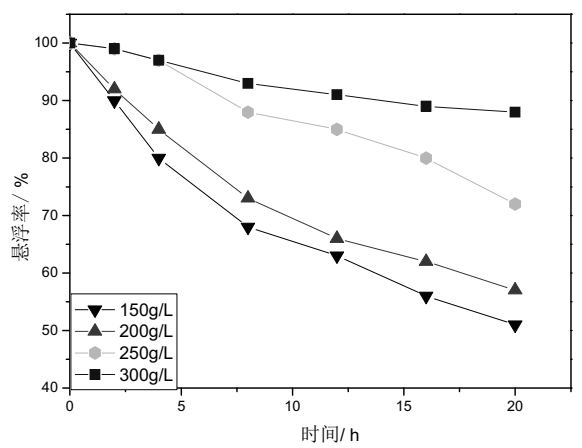

Fig. 3 Suspensibility curves of magnetic fluid prepared by PEG-4000 aqueous solution with different

concentration

When the concentration of PEG-4000 was less than $250 \mathrm{~g} / \mathrm{L}$, the suspension of magnetic fluid decreased with addition of PEG-4000 concentration. This was because, when the concentration of PEG-4000 was low, it could not form fully wrapping shell on the surface of the $\mathrm{Fe}_{3} \mathrm{O}_{4}$ nanoparticle, causing part of the $\mathrm{Fe}_{3} \mathrm{O}_{4}$ magnetic fluid exposed to the air, and so the suspension was decreased. The Fig shows when the PEG-4000 concentration rose to $300 \mathrm{~g} / \mathrm{L}$, the suspension was best, indicating that the PEG-4000 coated the $\mathrm{Fe}_{3} \mathrm{O}_{4}$ nanoparticles surface better at this concentration, preventing the agglomeration of nanoparticles. The magnetic fluid suspension changed for the better. The of $250 \mathrm{~g} / \mathrm{L}$ PEG aqueous solution and $300 \mathrm{~g} / \mathrm{L}$ PEG aqueous solution to synthesize the magnetic fluid had no large difference in suspension because the standing time was less than 8 hours. $250 \mathrm{~g} / \mathrm{L}$ was best PEG-4000 concentration for preparation of the magnetic fluid.

\section{6Influence of different concentrations for stability of MHD}

The optimal concentration for the PEG-4000 was $250 \mathrm{~g} / \mathrm{L}$. We compared the $\mathrm{pH} \approx 7$, effect of different concentrations for stability of the magnetic fluid, and suspension changes as shown in Fig. 4. 


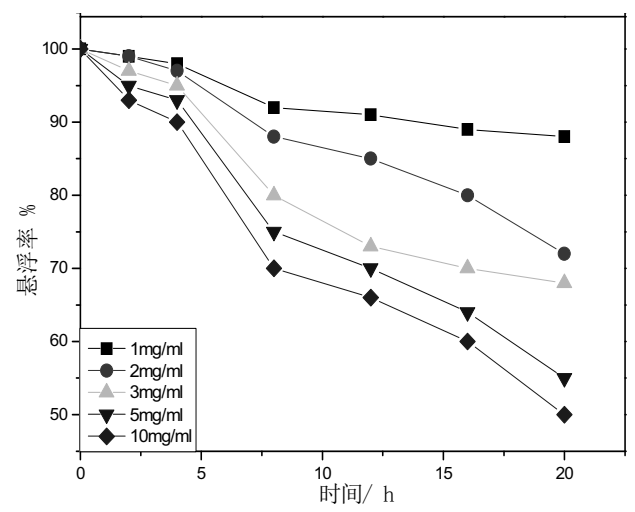

Fig. 4 Suspensibility curves of magnetic fluid with different concentration

As can be seen from Fig. 4, the suspension decreased with time while the concentration of magnetic fluid increased under the $\mathrm{pH}$ neutral conditions. The suspension property for the different concentrations of magnetic fluid was similar after standing for $2 \mathrm{~h}$, and all reached more than $90 \%$. There was clear change on the magnetic fluid suspension after standing for $8 \mathrm{~h}$ later. The concentration of the magnetic fluid suspension at $1 \mathrm{mg} / \mathrm{mL}$ was still more than $90 \%$ when allowed to stand for $20 \mathrm{~h}$, while the concentration at $10 \mathrm{mg} / \mathrm{mL}$ of magnetic fluid suspension dropped to about $50 \%$.

Subsidence caused the increased concentration of magnetic fluid in lower part during the standing of the magnetic fluid, reducing the upper concentration and then due to the Brownian motion, the formation of concentration gradient causes the reverse diffusion:

$$
\frac{\mathrm{d} n}{\mathrm{~d} z}=\frac{\pi d^{3}}{6 k T}\left(\rho-\rho_{*}\right) g n
$$

Formula (2-2) shows the conditions for stable suspension of the magnetic particles under gravity (where; $\mathrm{k}$ is Boltzmann's constant, $\mathrm{T}$ is the absolute temperature, $\mathrm{n}$ is the number of particles per unit volume, $\mathrm{z}$ is the height, $\mathrm{d}$ is the particle diameter, $\rho$ is the density of the particles, $\rho c$ is the density of the base liquid, and $g$ is the gravitational acceleration). As can be seen from the formula, the higher concentration caused the larger $\mathrm{n}$ and worsened the stability of the magnetic fluid. Potential is proportional to the volume of solid particles, meaning the higher the concentration, the greater the volume of the solid particles and the higher the potential for causing the worse stability. Therefore, the stability of the magnetic fluid was increased with decrease of the fluid mass concentration.

\subsection{Impact of different $\mathrm{pH}$ value of $\mathrm{MHD}$ stability}

For practical applications, the $\mathrm{pH}$ value for environmental change was large, so we also compared the suspension with $2 \mathrm{mg} / \mathrm{mL}$ concentration of magnetic fluid under different $\mathrm{pH}$ conditions, to investigate the variation of the magnetic fluid suspension with the $\mathrm{pH}$. The results are shown in Fig.5: 


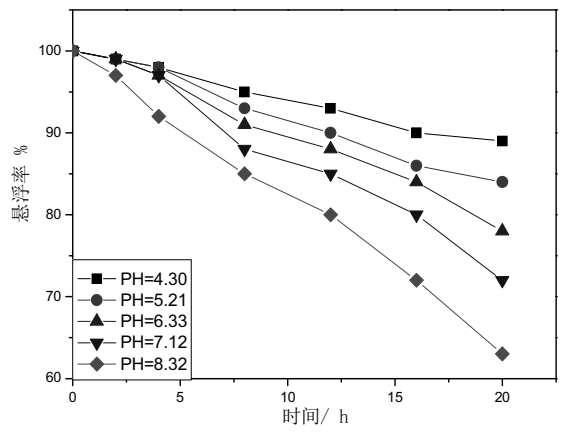

Fig. 5 Suspensibility curves of magnetic fluid at different PH value

Fig. 5 showed that the suspension of the prepared magnetic fluid under acidic conditions was better than that under neutral and basic conditions. The higher $\mathrm{pH}$ value caused the worse suspension. When the $\mathrm{pH}$ was equal to 8.32 , we could clearly see that the suspension of the magnetic fluid decreased rapidly. When the $\mathrm{pH}$ value of the magnetic fluid was adjusted, the main factor affecting the stability was the surface charge of particles. The electric double layer stabilization mechanism shows that the more the colloid surface charge, the greater the repulsive force between colloids, causing more stable colloids and slower particle settling velocity. The particle surface charge amount is proportional to the difference between the $\mathrm{pH}$ value of the solution and isoelectric point $\mathrm{pH}_{\text {iep }}$. Since the PEG -coated magnetic fluid $\mathrm{pH}_{\text {iep }}$ was about 10 , the $\mathrm{Fe}_{3} \mathrm{O}_{4}$ magnetic fluid $\mathrm{pH}$ value was thus in the range between 3-10.The amount of the surface charge decreased with the increase in the $\mathrm{pH}$, and therefore the stability of the magnetic fluid resistance decreased with the increase of $\mathrm{pH}$.

\section{Summary}

In this paper, water-based magnetic fluids $\left(\mathrm{Fe}_{3} \mathrm{O}_{4}\right)$ with good dispersion were synthesized by co-precipitation method. $\mathrm{FeCl}_{3} \cdot 6 \mathrm{H}_{2} \mathrm{O}$ and $\mathrm{FeCl}_{2} \cdot 4 \mathrm{H}_{2} \mathrm{O}$ were used as iron sources, while $\mathrm{NaOH}$ was used as precipitant and PEG-4000 as surfactant. The result from the XRD showed that the nanoparticles had better dispersion, and particle sizes were between 10-20 nm, proving them to be Nano $\mathrm{Fe}_{3} \mathrm{O}_{4}$. The water-based magnetic fluids had better dispersion both in ethanol and water when using the PEG-4000 as the surfactant. The nanoparticles could also be placed in the air for a long time, making them better for use in the preparation of core-shell structured composite materials when needed.

\section{References}

1. De-Cai Li. Magnetic liquid theory and application, Beijing: Science Press, 2003

2. Jiajie Liang, Yanfei $\mathrm{Xu}$, Dong Sui, et al, Flexible, Magnetic, and Electrically Conductive Graphene $/ \mathrm{Fe}_{3} \mathrm{O}_{4}$ Paper and Its Application for Magnetic-Controlled Switches, J. Phys. Chem. C 2010, 114, 17465-17471

3. P. D. Shima, John Philip,* and Baldev Raj, Synthesis of Aqueous and Nonaqueous Iron Oxide Nanofluids and Study of Temperature Dependence on Thermal Conductivity and Viscosity, J. Phys. Chem. C 2010, 114, 18825-18833

4. Reza Azizian, Elham Doroodchi, and Behdad Moghtaderi*, Effect of Nanoconvection Caused by Brownian Motion on the Enhancement of Thermal Conductivity in Nanofluids, Ind. Eng. Chem. Res. 2012, 51, 1782-1789

5. Gui-Ping Zhu, Nam-Trung Nguyen,* R. V. Ramanujan, and Xiao-Yang Huang, 
Nonlinear Deformation of a Ferrofluid Droplet in a Uniform Magnetic Field, Langmuir 2011, 27, 14834-14841

6. Katherine A. Mirica, Charles R. Mace, George M. Whitesides*, et al, Magnetic Levitation in the Analysis of Foods and Water, J. Agric. Food Chem. 2010, 58, 6565-6569

7. Mikhail V. Avdeev*, Birte Mucha, Katrin Lamszus, et al, Structure and in Vitro Biological Testing of Water-Based Ferrofluids Stabilized by Monocarboxylic Acids, Langmuir 2010, 26(11), 8503-8509 